\title{
Dry Eye Diseases and Ocular Surgery: Practical Guidelines for Canadian Eye Care Practitioners
}

for the Canadian Dry Eye Disease Consensus Panel

Paul M. Karpecki, OD, FAAO

Kentucky Eye Institute, University of Pikeville School of Optometry

\section{Lisa Prokopich, OD, MSc} University of Waterloo, School of Optometry and Vision Science

Louis Racine, MD, FRCSC Université de Montréal, Faculté de médecine, Département d'ophtalmologie

Etty Bitton, OD, MSc, FAAO Université de Montréal, École d'optométrie

Barbara Caffery, OD, PhD

Toronto Eye Care

Paul Harasymowycz, MD, FRCSC

Montreal Glaucoma Institute

Langis Michaud, OD, MSc, FAAO

Université de Montréal, École d'optométrie

Victor D. Pegado, MD, FRCSC, Dipl. ABO University of British Columbia, Faculty of Medicine, Department of Ophthalmology and Visual Sciences

Jean-Sébastien Dufour, OD, MSc

Université de Montréal, École d'optométrie

Paul Neumann, OD Central Saanich Optometry

Andrew Webber, OD Elmsdale Vision Centre

John Ashkenas, PhD SCRIPT

\section{ABSTRACT:}

In 2014, the Canadian Dry Eye Disease Consensus Panel published Guidelines for screening, diagnosis and management of dry eye diseases (DED). These did not address the implications of DED for individuals who are being considered for or have recently undergone ocular surgery. DED is common in certain surgical cohorts, and the perisurgical setting poses specific challenges, both because surgery can complicate preexisting DED and because symptomatic and non-symptomatic DED place the patient at risk of poor surgical outcomes. The Consensus Panel has developed this Addendum to the 2014 Guidelines to offer guidance on DED care before and after ocular surgery.

\section{SHORT TITLE:}

Perisurgical Dry Eye Disease

\section{CORRESPONDING AUTHOR:}

Paul M. Karpecki, OD, FAAO, Kentucky Eye Institute, 601 Perimeter Dr., Ste. 100, Lexington KY 40517, Tel: 859 278-9393, Fax: 859 277-3965, E-mail: paul@karpecki.com

KEY WORDS:

Dry eye disease, keratoconjunctivitis sicca, phacoemulsification, LASIK, quality of vision, ocular neuropathic pain 


\section{INTRODUCTION}

\section{Dry Eye Disease and the 2014 Canadian Guidelines}

Dry eye disease (DED), also known as keratoconjunctivitis sicca, comprises a group of inflammatory ocularsurface diseases that, collectively, are ubiquitous ${ }^{1,2}$ in the general population. DED is highly prevalent in specific subpopulations, including adults over age $50,{ }^{1}$ women, people with long-standing diabetes, ${ }^{3}$ and people who wear contact lenses. ${ }^{4,5}$ Rarer conditions associated with severe DED include Sjögren syndrome (SS), ${ }^{6}$ Steven-Johnson syndrome, nocturnal lagophthalmos, exposure keratopathy, and graft-versus-host disease., ${ }^{7,8}$

DED encompasses multiple conditions in which the precorneal tear film is scarce, unstable, inadequately distributed, or of abnormal composition. For instance, tears may be deficient in lipids (Meibomian gland dysfunction, a common cause of evaporative DED), water (e.g., in SS), or mucins (e.g., due to genetic deficiency or loss of conjunctival goblet cell function). ${ }^{8}$ Tears in DED become hyperosmolar and may accumulate cytokines that trigger and perpetuate local inflammation. Hyperosmolar tears can damage the corneal and conjunctival epithelia, in part because they carry high levels of inflammatory mediators, such as the degradative enzyme matrix metalloproteinase-9. ${ }^{9}$

DED may present with any combination of ocular dryness, fatigue, redness, burning, itching or stinging pain, foreign-body sensation, and light sensitivity, as well as mucus filaments, eyelid irritation and crusting. Patients may report blurred or fluctuating vision and may experience reduced visual acuity and impaired functioning in visual tasks. Use of dyes for ocular-surface staining during routine exams often detects conjunctival and corneal abnormalities such as superficial punctate keratitis. ${ }^{10}$ In extreme cases, DED can lead to corneal ulceration, neovascularization, permanent scarring, and irreversible visual loss. ${ }^{11}$

\section{OCULAR NEUROPATHIC PAIN}

Patients who suffer from neuropathic eye pain may describe their subjective experience in terms similar to those used for DED. Common pain descriptors used for both conditions include "burning," "sharp," and "gritty," and both can cause light sensitivity. This similarity of presentation frequently leads to confusion and treatment dissatisfaction, particularly for individuals who develop persistent or intractable postsurgical pain. ${ }^{24,89}$

The origins of neuropathic pain are obscure, but nerve damage from various sources may be a proximate cause. ${ }^{23,34,90}$ Aberrant nerve regeneration and several other central and peripheral neurologic events ${ }^{22}$ have been proposed to reinforce the neuropathy, causing the pain to become chronic. ${ }^{24,89}$

Eye pain may be identified for the first time as neuropathic following surgery. However, neuropathic ocular pain is also widely found in patients with no history of ocular surgery. Pain reported after surgery sometimes follows a pre-existing pattern that might have been misattributed to DED or other causes. A neuropathic origin should be suspected when ocular hypersensitivity, hyperalgesia (exaggerated pain response to suprathreshold noxious stimuli), or allodynia (pain in response to normally non-noxious stimuli) are not commensurate with objective signs of DED, such as corneal and conjunctival staining.

This possibility can be tested by instilling anesthetic drops, thus blunting nociceptive signals from corneal neurons. ${ }^{22,24}$ By definition, neuropathic pain originates in the brainstem or elsewhere in the central nervous system. Therefore, although this test is not diagnostic, the observation of pain that persists despite topical anesthesia is suggestive of neuropathy. Neuropathic pain is also reported to be poorly responsive to artificial tears, relative to physiologic (nociceptive) pain. ${ }^{91}$

Common comorbidities include general (non-ocular) neuropathic pain, as well as depression, anxiety, and sleep disturbance, ${ }^{22,92,93}$ patients reporting otherwise-unexplained eye pain should be queried about these other conditions as well. 
In 2014, the Canadian Dry Eye Disease Consensus Panel presented Guidelines on DED screening, diagnosis and management. ${ }^{8}$ This guidance (Fig. 1) offered general principles for diagnosing and managing episodic, chronic, and recalcitrant DED and for monitoring the effectiveness of treatment. Based on these Guidelines, it is essential for all eye care professionals to screen for ocular-surface diseases and manage them appropriately. As discussed in the Guidelines, episodic DED can sometimes be managed with lubricating eye drops, eyelid hygiene, and/or modifications to the living and working environment. Conversely, chronic DED is an inflammatory disease that requires anti-inflammatory treatment. ${ }^{8}$ Currently available options to manage ocular-surface inflammation include topical corticosteroids, which are generally reserved for short-term use, as well as essential fatty acids and cyclosporine $0.05 \%$ emulsion in castor oil (Restasis ${ }^{\varpi}$, Allergan Inc.; throughout this Addendum, cyclosporine refers to this formulation). ${ }^{8}$ Additional topical anti-inflammatory products, such as lifitegrast $5 \%$ and cyclosporine $0.1 \%$ in a cationic formulation, may soon reach the Canadian market for use in cases of DED. ${ }^{12-17}$ To date, there have been no reports on the perisurgical use of these additional products.

Figure 1: Diagnosis and management of DED, according to the 2014 Canadian Guidelines ${ }^{8}$

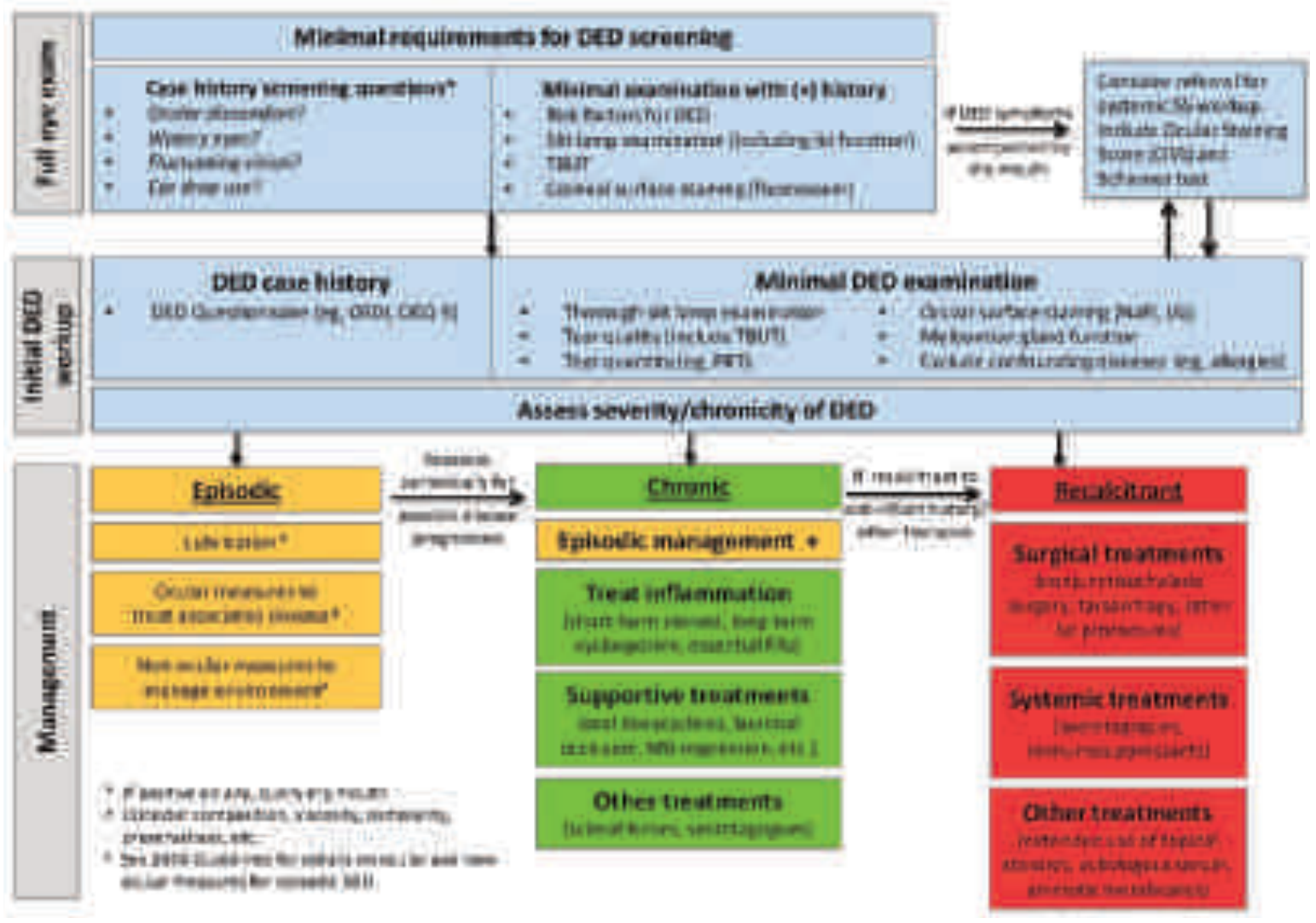

\section{Scope and Aims of this Addendum}

The 2014 Guidelines did not address DED management in individuals undergoing ocular surgery. This topic is significant because of the variety of ways that uncontrolled DED can become problematic for patients undergoing procedures such as cataract surgery or refractive surgery. In addition, ocular surgeries of many types (Table 1) can induce ocular-surface inflammation, which results from direct trauma to the cornea, light toxicity from the surgical microscope, tear-film evaporation during surgery, and irritation due to topical anesthetics, surface antiseptic solutions, and preservative-containing eye drops. ${ }^{18-21}$ As a result, surgery can precipitate de novo DED or exacerbate the condition in patients with pre-existing symptomatic or asymptomatic DED. Postsurgical ocular dryness and pain that present during the recovery phase are usually transient, but can persist in certain individuals. Chronic pain without significant ocular-surface disease ("pain without stain") 22 can also occur, and may be present before or after surgery; such neuropathic eye pain, resulting from lesions within the somatosensory nervous system, ${ }^{23}$ is not readily distinguished from DED. This confusion poses a variety of clinical problems that can be frustrating for both patients and caregivers (see sidebar on Ocular Neuropathic Pain). ${ }^{22,24}$ 
As discussed below, pre-existing ocular-surface disease complicates presurgical biometry, keratometry, topography and refraction, thus increasing a patient's risk of unsatisfactory visual correction and of refractive regression. DED can degrade the quality of vision (QoV) after surgery, leading to discomfort and dissatisfaction, even among patients whose visual acuity has been corrected effectively. Conversely, measures that restore the tear film, reduce tear osmolarity, or suppress corneal and conjunctival inflammation may improve postsurgical DED symptoms and visual outcomes. Thus, there are several compelling reasons to manage DED, both before and after surgery. Nevertheless, in surgical candidates and others, ocular-surface disease frequently goes unrecognized and untreated. ${ }^{8,25}$ It is crucial for eye care professionals to recognize DED signs and symptoms in surgical candidates, to begin treatment promptly, and wherever possible, to reverse ocular-surface inflammation before proceeding to surgery.

Table 1: Ocular surgeries that may require DED diagnosis and management

\begin{tabular}{|c|c|c|c|c|}
\hline Type of surgery & $\begin{array}{l}\text { Surgery induces or } \\
\text { complicates DED? }\end{array}$ & $\begin{array}{l}\text { DED complicates } \\
\text { surgery? }\end{array}$ & $\begin{array}{l}\text { DED highly } \\
\text { prevalent in surgical } \\
\text { cohort? }\end{array}$ & References \\
\hline \multicolumn{5}{|l|}{ Refractive } \\
\hline LASIK & Yes & Yes & Yes & This article \\
\hline PRK & Yes & Yes & Yes & 110 \\
\hline \multicolumn{5}{|l|}{ Cataract } \\
\hline Phacoemulsification & Yes & Yes & Yes & This article \\
\hline \multicolumn{5}{|l|}{ Corneal/conjunctival } \\
\hline Penetrating keratoplasty & Yes & Yes & Yes & ${ }^{111-113}$ \\
\hline Endothelial keratoplasty & Yes & Yes & Yes & $111-114$ \\
\hline Pterygium excision & Yes & No & Yes & 115 \\
\hline Conjunctivochalasis removal & No & Yes & Yes & $116-119$ \\
\hline \multicolumn{5}{|l|}{ Vitreo-retinal } \\
\hline Vitrectomy & Yes & $?$ & Yes & 120,121 \\
\hline \multicolumn{5}{|l|}{ Eyelid } \\
\hline Blepharoplasty & Yes & No & Yes & $122-124$ \\
\hline Ptosis repair & Yes & $?$ & No & 122 \\
\hline \multicolumn{5}{|l|}{ Glaucoma } \\
\hline Trabeculectomy & Yes & Yes & Yes & 125,126 \\
\hline \multicolumn{5}{|l|}{ Others } \\
\hline Strabismus repair & Yes & No & No & 127 \\
\hline
\end{tabular}

Therefore, we developed this Addendum to the 2014 DED Guidelines for Optometrists specifically to address the perisurgical management of DED. The focus here is on cataract surgery, mainly phacoemulsification with intraocular lens (IOL) implantation and refractive procedures such as laser-assisted in situ keratomileusis (LASIK). Comorbid DED in glaucoma is discussed in the sidebar on Dry Eye Disease in Glaucoma. 
DRY EYE DISEASE IN GLAUCOMA

DED is a common comorbidity with glaucoma, occurring in $15 \%$ to $59 \%$ of glaucoma patients. ${ }^{94-99}$ This association can be explained at least in part by medicated eye drop polypharmacy, and specifically by the presence and duration of exposure to irritants found in medicated and non-medicated products, including active pharmaceutical ingredients and preservatives such as benzalkonium chloride (BAK)..$^{99,100}$

Inflammation in response to BAK may be heightened by the presence of hyperosmolar tears, ${ }^{101}$ as is common in DED. Moreover, chronic use of BAK-containing products can lead to increased tear-film osmolarity, and DED can complicate ophthalmic surgery, including procedures for glaucoma. ${ }^{94}$ In patients using BAKcontaining products, a preoperative course of topical steroids may decrease conjunctival inflammation and increase the rate of successful trabeculectomy; of course, it is essential to monitor intraocular pressure if steroids are used. ${ }^{102,103}$

Preservative-free artificial tears (ATs) are associated with reduced DED symptoms ${ }^{97}$ and should be used in preference to preserved ATs. ${ }^{8}$ However, it is rarely feasible for patients with glaucoma to avoid the use of irritating topical medications, especially as the disease progresses and topical monotherapy must be abandoned. ${ }^{100,104,105}$ For this reason, anti-inflammatory treatment may be considered, to ameliorate DED in patients requiring long-term exposure to topical glaucoma medications. ${ }^{106}$ Whereas it has been generally assumed that controlling glaucoma is the primary goal when these two conditions co-exist, recent findings suggest that surface optimization in patients with comorbid glaucoma is compatible with reducing intraocular pressure. ${ }^{107,108}$

Trabeculectomy and other surgeries for glaucoma temporarily exacerbate DED, but offer the prospect of long-term IOP control with greatly reduced need for topical glaucoma medications. Indeed, $40 \%$ of patients no longer require any topical glaucoma medications for up to 3 years post-trabeculectomy. ${ }^{109}$ With the advent of less-invasive surgical procedures, it may be possible to allow for better control of IOP at an earlier point in the progression of glaucoma, thus reducing the chronic exposure to irritating topical medications. So-called micro-invasive glaucoma surgeries (MIGS) are $a b$ interno procedures, meaning that they are carried out from inside the eye and cause minimal or no trauma to the conjunctiva. MIGS procedures are considered sufficiently low risk that their use can be justified even in individuals with mild to moderate glaucoma. ${ }^{96}$ Although direct evidence is still lacking, MIGS procedures are therefore expected to carry less risk of inducing DED, compared to traditional surgery.

The general DED management approaches described in the Canadian Guidelines are considered to be applicable to patients requiring surgery. For this reason, this Addendum focuses on questions that are specific to perisurgical DED care, such as:

\begin{abstract}
What evidence implicates uncontrolled DED in adverse post-surgical outcomes? How should the presence of DED affect decisions on the timing or appropriateness of a procedure? How should DED be managed before and after a procedure?

How should optometrists and ophthalmologists co-manage DED in individuals being considered for ocular surgery?
\end{abstract}

\title{
DED, VISUAL FUNCTIONING, AND OCULAR BIOMETRY
}

The precorneal tear film, the first refractive surface of the eye, functions best when mirror-smooth. The tear film is maintained by neuroendocrine mechanisms that regulate secretory function and the blink rate in response to shifting environmental stresses. ${ }^{10,26,27}$ A healthy tear film is sufficiently thick, uniform, and balanced with appropriate components to protect the ocular surface from insult and to avoid optical aberrations between blinks. ${ }^{28-31}$

Abnormalities in the tear film, affecting either tear quantity or composition, can lead to aqueous-deficient or evaporative ocular-surface diseases. Surgical trauma compromises tear-film regulation, at least temporarily. For example, loss of tactile sensation at surgically denervated sites in the cornea impairs basal and reflex tearing and reduces the blink rate, leading to a compromised tear film and ocular surface while the damaged nerves regrow. ${ }^{27,32-34}$ 
DED affects QoV without necessarily degrading visual acuity. ${ }^{1,29,35}$ For instance, DED is associated with glare, impaired contrast sensitivity and symptoms of higher order aberrations (HOAs); patients experience fluctuating vision during the interval between successive blinks, due to a non-uniform and unstable tear film. ${ }^{27}$ QoV is an important predictor of daily function, especially for tasks that place high demands on the visual system such as reading or driving. ${ }^{36,37}$

In patients undergoing ocular surgery, visual disturbances related to DED may increase the risk of postsurgical patient dissatisfaction. For both refractive procedures and cataract surgery with IOL implantation, surgical planning requires accurate keratometry and/or topography. Uncontrolled DED can alter the shape of the cornea, ${ }^{38}$ reducing the accuracy and precision of biometric findings. Tear hyperosmolarity is associated with greater statistical scatter in keratometric readings. Ocular-surface irregularity and instability reduce the precision of preoperative IOL and refractive calculations, increasing the risk of suboptimal refraction following surgery, especially when toric or multifocal implants are used. ${ }^{39}$ In addition, even for individuals who achieve good postsurgical acuity, DED can reduce QoV over a period of weeks to months, and sometimes longer. ${ }^{40}$

Table 2 summarizes various goals for DED treatment in the general population and in candidates for ocular surgery.

Table 2: Goals of DED management

- To ameliorate eye discomfort and fatigue

- To prevent corneal erosion and surface anomalies associated with ocular-surface disease and tear hyperosmolarity ${ }^{3,28,32,39}$

- To prevent optical aberrations that reduce visual quality (e.g., blurring, glare, loss of contrast sensitivity $)^{30,35}$

- To improve performance and facility in demanding visual tasks (e.g., reading ${ }^{36}$ and driving ${ }^{37}$ )
- To achieve more accurate and precise biometric/ keratometric refractive measurements, allowing IOL power or LASIK surface parameters to be calculated more confidently

- To improve postsurgical visual acuity and quality of vision

- To prevent or minimize postsurgical $\mathrm{DED}^{40}$

\section{The Growing Need for Collaborative Care for DED}

Several demographic and societal trends have increased both the urgency and the burden of managing DED. First, both DED and ocular surgeries are increasingly common as the population ages. Second, the increasing reliance on and use of electronic devices can cause or exacerbate DED by decreasing the user's blink rate. ${ }^{41}$, ${ }^{42}$ People who use these devices require a high level of visual functioning and may seek surgical intervention specifically to improve their QoV. Third, ophthalmic technology itself has changed with the introduction of measurement approaches that offer unprecedented precision in ocular biometry but that rely on a healthy tear film if they are to be used optimally. Likewise, multifocal and toric IOLs offer the prospect of corrected near and distance vision, but they appear to be more sensitive than earlier-generation IOLs to both visual aberrations and errors in biometry. ${ }^{43}$ This difference is intrinsic to the IOL technology, but dissatisfaction also results in part from higher expectations, including the desire for optimal visual functioning with minimal dependence on distance or reading glasses.

For all of these reasons, the growing need for diligent pre- and postsurgical DED care is placing increasing demands on caregivers' time. Efficient approaches, including collaborative DED management by optometrists and ophthalmologists, will be needed to meet these demands.

\section{CATARACT SURGERY}

If we extrapolate from 2014 data from a single province, approximately 450,000 phacoemulsification procedures may be carried out annually across Canada, making cataracts one of the most common reasons for ocular surgery. ${ }^{44,45}$ The demand for this surgery is projected to more than double by $2036 .{ }^{46}$

The current standard of care for cataract extraction is phacoemulsification followed by implantation of an IOL, which may be either monofocal or premium. The latter type of IOL includes a variety of designs that allow for correction of distance, reading, and intermediate vision, generally resulting in less dependence on glasses or contact 
lenses. Depending on their design, premium IOLs may be prone to glare and halos, so it is important to minimize other visual disturbances in these patients, including aberrations related to DED. ${ }^{47}, 48$ Interestingly, cataracts themselves can induce HOAs, ${ }^{49,50}$ and this effect on QoV is complicated by poorly controlled DED. ${ }^{29,30}$

Pre-existing DED is common among individuals with cataracts, since some risk factors (notably advancing age, diabetes $^{3}$, and female sex ${ }^{51}$ ) predispose patients to both conditions. ${ }^{52}$ However, DED commonly goes unrecognized and untreated in patients undergoing cataract surgery. Trattler et al. reported that, out of 136 American patients undergoing cataract surgery, while only $22 \%$ had a prior diagnosis of DED, a larger proportion showed objective signs of surface disease (tear break-up time (TBUT) $\leq 5$ seconds, $63 \%$; corneal staining, $77 \%$ ) suggesting widespread underdiagnosis. ${ }^{25}$ Interestingly, subjective symptoms of DED were less common, with only $31 \%$ reporting stinging and $41 \%$ reporting foreign-body sensation. These findings are consistent with previous reports suggesting that patient self-reporting is an unreliable screening tool for DED. ${ }^{41,53,54}$

Uncontrolled DED limits the accuracy of preoperative biometry, leading to errors in IOL power or placement. ${ }^{41}$, ${ }^{54}$ This effect has been clearly demonstrated using repeated readings in patients presenting for cataract surgery. Epitropoulos et al. reported that the mean difference between two successive keratometric readings was $0.28 \mathrm{D}$ among individuals with hyperosmolar tears ( $\mathrm{n}=100$ eyes) versus $0.13 \mathrm{D}$ among controls ( $\mathrm{n}=50$ eyes). Calculated IOL power differences were up to $5.5 \mathrm{D}$ among 100 eyes with hyperosmolar tears, and the frequency of an IOL power difference $\geq 0.5 \mathrm{D}$ was significantly higher with hyperosmolar versus normal tears $(\mathrm{p}=0.02)$. In addition, $17 \%$ of eyes with hyperosmolar tears but only $2 \%$ of eyes with normal tears showed a vector astigmatism difference $\geq 1.0 \mathrm{D}$ $(\mathrm{p}=0.01)$ between readings. ${ }^{39}$

\section{Onset of Symptoms of DED Following Cataract Surgery}

Cataract surgery perturbs the ocular surface and induces intraocular and ocular-surface inflammation. In addition, the surgical procedure damages sensory and other neurons, and the resulting denervation reduces tactile and other sensation in the cornea. ${ }^{40}$ De novo DED symptoms are common following phacoemulsification, ${ }^{10}$, ${ }^{27}$ but they are usually transient. Corneal hypoesthesia, tear-film instability, and other indicators of DED often resolve within 3 months, probably associated with the beginning of axonal regrowth. ${ }^{18,19}$ Corneal sensation gradually returns to near-preoperative levels over the course of 1 year. ${ }^{40}$ In a small subset of patients, however, DED symptoms persist indefinitely. ${ }^{20,55}$ For instance, individuals with diabetes are at increased risk of severe and chronic postsurgical DED. ${ }^{56}$

Topical treatments should be applied consistently following surgery to limit the extent or duration of de novo DED. ${ }^{52}$ Used alongside topical steroids, lubricants have been reported to improve symptoms of DED and visual functioning, relative to standard postsurgical topical care alone. ${ }^{57,58}$ Jee et al. directly compared the effects of preservative-free versus preserved steroid and lubricant eye drops after cataract surgery in 80 patients (80 eyes) with pre-existing DED. In this prospective, open-label study, patients received the preservative-free or preserved products 4 times daily for 1 month and twice daily thereafter. By Month 1 following surgery, subjects who received the preservative-free topical treatment reported significantly less severe symptoms compared to those who received preserved treatment $(\mathrm{p}<0.05)$. By Month 2, objective DED measures (staining, tear-film stability, inflammatory markers, and conjunctival goblet cells) were significantly improved with preservativefree treatment. ${ }^{59}$

Lubrication alone may be insufficient to manage the inflammation that drives chronic DED. ${ }^{60-62}$ This has been shown most clearly in a randomized, multi-centre study of 233 Chinese adults with moderate to severe DED at baseline. Patients were randomized to twice-daily application of cyclosporine $0.05 \%$ or the emulsion that serves as its vehicle, with no other treatment permitted except for artificial tears. Whereas both groups experienced significant symptomatic improvement over baseline, significantly greater improvement was seen in corneal staining at 4 and 8 weeks $(p=0.025$ and 0.05 , respectively) and in the Schirmer score at 4 weeks $(\mathrm{p}=0.035)$ with cyclosporine versus vehicle. ${ }^{60}$ While no such vehicle-controlled study has been reported in a surgical setting, a prospective, contralaterally controlled study compared topical cyclosporine with saline in 32 patients undergoing bilateral phacoemulsification. In these patients, treatment with topical cyclosporine significantly improved tear-film stability and other measures of DED, relative to saline alone. While patientreported DED intensity improved by the first month of treatment, clinical benefits became statistically significant by 2 months of cyclosporine treatment. ${ }^{63}$ 


\section{Exacerbation of DED after Cataract Surgery}

For patients with pre-existing chronic DED, anti-inflammatory treatment may improve postsurgical acuity and visual functioning. ${ }^{47,64}$ In a small, prospective, contralaterally controlled, randomized, double-masked study, Donnenfeld et al. compared lubricating eye drops ( $0.4 \%$ polyethylene glycol $400 ; 0.3 \%$ propylene glycol) to topical cyclosporine in patients undergoing bilateral phacoemulsification with multifocal IOL implantation. Of the 14 individuals studied, only 3 had been diagnosed as DED. However, because the mean baseline TBUT was low (approximately 6 seconds), others may have had undiagnosed or marginal DED. Treatment was initiated 1 month before surgery and maintained after the procedure. By Month 2 following surgery (i.e., after 3 months of topical treatment), uncorrected visual acuity was significantly better for eyes treated with cyclosporine than with lubricants alone $(\mathrm{p}=0.005)$. Contrast sensitivity was also improved with cyclosporine treatment, and there was a numeric trend toward greater tear stability at Month 2. Furthermore, corneal staining, which worsened from baseline in lubricant-treated eyes, improved significantly in cyclosporine-treated eyes $(\mathrm{p}=0.034$ for the between-group difference at Month 2$){ }_{{ }^{47}}$

Other studies have explored the postsurgical use of topical cyclosporine in cataract surgery. ${ }^{63-65}$ It has been suggested that some treatment effects are seen within weeks of phacoemulsification ${ }^{65}$ and other surgeries, ${ }^{66}$ but these claims are difficult to evaluate, given that the established benefits of cyclosporine occur with longer-term use ( $\geq 3$ months). ${ }^{8,54,61}$

\section{REFRACTIVE SURGERY}

LASIK and related photorefractive procedures are widely used to improve uncorrected visual acuity. These procedures generally lead to favorable outcomes and high patient satisfaction. ${ }^{67}{ }^{68}$ However, postoperative dry eye is a possible complication of these procedures and a cause of discomfort, reduced vision, and overall dissatisfaction. As with cataract surgery (above), DED following LASIK is associated with the effects of surgical trauma on the ocular surface, including loss of sensation in the cornea ${ }^{40}$ and suppression of blinking and both reflex and basal tearing. ${ }^{69}$ Pre-existing DED is likely common and underdiagnosed, given that contact lens intolerance, which has been linked to DED, is a common motivator for patients requesting LASIK. ${ }^{67}$

\section{Dry Eye Disease as a Factor in Patient Selection}

Because refractive surgeries are elective, only individuals who have or can attain adequate ocular-surface health are considered candidates., ${ }^{232,70,71}$ Severe DED due to SS or other immune causes is usually considered a contraindication for surgical refractive treatment. However, some reports suggest that even these individuals may be candidates for LASIK if the condition is successfully managed before the procedure. ${ }^{68,72}$ To this end, pre-existing DED should be managed in a stepwise manner (Fig. 1), using therapies described in the 2014 Guidelines. ${ }^{8}$ Notably, one retrospective study found that cyclosporine treatment for an average of 3.2 months (range 1-12 months) enabled those with mild DED to proceed with refractive surgery. ${ }^{73}$

Alternative photorefractive surgeries, including flapless procedures such as small-incision lenticule extraction (SMILE), appear to cause less nerve damage and less severe DED than does LASIK.74,75 Whether these newer procedures are preferable for patients at high risk of DED has not yet been established. ${ }^{69,72}$

\section{Transient versus Chronic DED Following LASIK}

Following LASIK, tear-film instability and other DED signs and symptoms usually resolve spontaneously, but chronic postsurgical DED, persisting 6 to 12 months after LASIK, has been reported in $0.8 \%$ to $20 \%$ of patients. ${ }^{70,76}$ The risk of chronic post-LASIK DED increases with age and is greater in women than in men.71,73 It has also been suggested that hyperopic LASIK procedures carry a greater risk of chronic DED than does myopic LASIK.73,77,78 Following hyperopic LASIK, patients with pre-existing DED and those who develop chronic DED appear to be at high risk of refractive regression..$^{70,77}$

In addition, pre-existing mild or subclinical DED appears to be a risk factor for chronic post-LASIK DED. ${ }^{32}$ One prospective study followed 139 eyes undergoing LASIK. Subjects were excluded if they had a definitive diagnosis of DED, but could be included with isolated symptoms such as mild or moderate corneal staining or TBUT $<5$ seconds. In this population, presurgical corneal staining and low tear production were associated with DED persisting 1 year after LASIK. ${ }^{76}$ Similarly, patients with reduced tear-film stability at baseline were at significantly greater risk of developing surface abnormalities over at least the first 6 months after LASIK. ${ }^{79}$

Use of topical cyclosporine following LASIK has been explored as a possible supplement to standard post-procedural care for patients with no prior diagnosis of DED. Peyman et al. reported that, in a series of 22 bilaterally 
treated subjects, eyes receiving daily cyclosporine were significantly more likely to recover tactile sensitivity within 3 months following LASIK, relative to control eyes $(\mathrm{p} \leq 0.011){ }^{80}$ The timing of this response is consistent with evidence that the clinical benefits of cyclosporine become significant after $\geq 3$ months of treatment., ${ }^{81,66}$

Post-LASIK visual acuity is less satisfactory among patients who develop chronic DED relative to patients who have no such adverse effect..$^{70,77,78}$ In a large $(n=565)$ retrospective analysis, Albietz et al. reported that refractive regression was associated with chronic DED ( $\mathrm{p}=0.008$ for DED at Month 12 vs no DED) and with pre-existing ocular-surface staining, reduced TBUT, and reduced corneal tactile sensation. ${ }^{81}$ The authors noted that DED in their patients abated with persistent ocular-surface management, involving placement of lacrimal occlusion (punctal plugs), lid hygiene, and consistent use of lubricating eye drops. In eyes with evidence of post-LASIK DED, uncorrected visual acuity and DED signs and symptoms improved in parallel, ${ }^{81}$ as has been reported outside the surgical setting. ${ }^{82}$

Taken together, these findings suggest that individuals at risk of post-LASIK DED benefit from topical treatments that restore the tear film and target inflammation.

\section{Exacerbation of DED after LASIK}

Salib et al. tested pre-LASIK DED treatment using cyclosporine versus unpreserved artificial tears in patients with a history of DED ( $\mathrm{n}=21$ [42 eyes]). ${ }^{83}$ Those scheduled to undergo myopic LASIK began twice-daily treatment with the randomized drops 1 month before the procedure. For both of these treatments, corneal-surface staining and subjective DED symptoms improved by the time of the procedure. Topical treatment was maintained over the following 3 months. DED symptoms worsened transiently at the Week 1 observation, with faster recovery seen in the cyclosporine-treated eyes relative to eyes treated with artificial tears. From 3 to 12 months following LASIK, DED symptoms improved relative to the baseline evaluation in both treatment groups. Cyclosporinetreated eyes showed greater predictability in refraction over the l-year observation period and were more likely to be within $0.5 \mathrm{D}$ of their target spherical equivalent refraction. This difference was statistically significant by Month $3(\mathrm{p}=0.015){ }^{83}$

If cyclosporine is to be used in individuals being considered for LASIK, treatment should begin $\geq 1$ month before the procedure and should be maintained for $\geq 3$ months afterwards, to maximize the benefit when DED symptoms are most troublesome. Pretreatment may allow individuals who would not otherwise be considered for refractive surgery to undergo LASIK successfully, ${ }^{32}$ and should be considered for those with evidence of mild or asymptomatic DED. ${ }^{66,80}$

In addition to anti-inflammatory treatment, ${ }^{66,80,83}$ pre- and post-LASIK therapeutic options include a broad range of options discussed in the 2014 Guidelines, ${ }^{8}$ such as lubricating eye drops, scleral lenses, lacrimal occlusion, essential fatty acid supplementation, ${ }^{84,85}$ autologous serum, and oral doxycycline. Perisurgical data on most of these options are highly limited. In one small study of 12 individuals with residual refractive errors after LASIK whose uncorrected vision improved with the application of lubricating artificial tears, punctal plugs appeared to improve visual performance significantly $(\mathrm{p}<0.0001){ }^{86}$ In addition, Di Pasquale et al. suggested that patients showing continued tear-film instability after ocular-surface inflammation has been addressed may benefit from an eye-warming procedure, ${ }^{87}$ to stimulate Meibomian gland secretion and restore tear lipids. ${ }^{8}{ }^{88}$ In an observational study on patients whose de novo DED persisted for 1 year after LASIK, the combination of punctal plugs, topical corticosteroids, and, where indicated, warm compresses, led to subjective symptomatic improvement in DED, as well as significant increases in tear lipid thickness and TBUT. ${ }^{87}$

\section{COLLABORATIVE MANAGEMENT OF DED}

Table 3 lists a series of goals for pre- and postoperative treatment of patients who are being considered for ocular surgery. The list is general, in that it could apply equally to cataract and refractive surgery and potentially to other procedures whose outcomes can be compromised by uncontrolled DED. Briefly, all patients who are referred for surgery consultation should be assessed carefully for symptoms and signs of ocular-surface disease. Patients with episodic or chronic DED should be treated prior to surgery with a goal of addressing both the signs and symptoms of DED and stabilizing the tear film and the ocular surface, to ensure that keratometric and topographic data are reliable and precise. Appropriate counseling during this treatment period is also critical to improve the patient's understanding of the condition and to manage expectations. After the procedure, ocular-surface disease may develop or worsen, at least transiently, despite the continued use of DED therapies initiated before surgery. The goal of care during this period should be to manage ocular discomfort and to restore a stable and healthy ocular surface. 
Table 3: Goals for pre- and post-surgical management of DED

\begin{tabular}{|l|l|}
\hline In the preoperative setting, achieve: & In the postoperative setting: \\
\hline 1. Minimal DED signs on exam & 1. Maintain presurgical treatment if ocular surface is stable \\
\hline 2. Minimal and controlled discomfort & $\begin{array}{l}\text { 2. Otherwise, intensify treatment until ocular surface } \\
\text { is stable and returns to an adequate baseline }\end{array}$ \\
\hline 3. Stable, optimized tear film & \\
\hline 4. Stable keratometric readings (biometric and topographic) & \\
\hline 5. Stable manifest refraction & \\
\hline
\end{tabular}

While the locus of care before and after surgery may vary somewhat based on the circumstances, in general, optometrists are well-placed to deliver much of the DED care that patients will require before and after surgery. DED is often detected first while the patient is under an optometrist's care. Whether or not the patient is being considered for ocular surgery, the optometrist should assess the ocular surface and initiate appropriate treatment without delay. In all cases, the role of the tear film and ocular surface needs to be addressed with surgical candidates, to reinforce the need for good treatment adherence and to avoid postsurgical disappointment. If premium IOLs are being considered, the patient should be informed that these devices are particularly sensitive to ocular-surface disturbance.

Figure 2 shows a schema for the efficient co-management of patients requiring or requesting ocular surgery, who have been diagnosed with symptomatic or asymptomatic DED. In such cases, the optometrist should describe in a referral letter to the surgeon all ocular-surface findings, including subjective reports and objective evidence leading to this diagnosis. Ideally, the optometrist will manage the condition at this early point, both to improve the patient's immediate comfort and visual function and to streamline management of the surgery. The optometrist's correspondence should describe DED tests and treatments to date, as well as the outcome of these treatments. If appropriate, the optometrist may also recommend that the procedure be delayed to allow time to optimize the ocular surface.

Figure 2: Proposed schema for the co-management of perisurgical DED
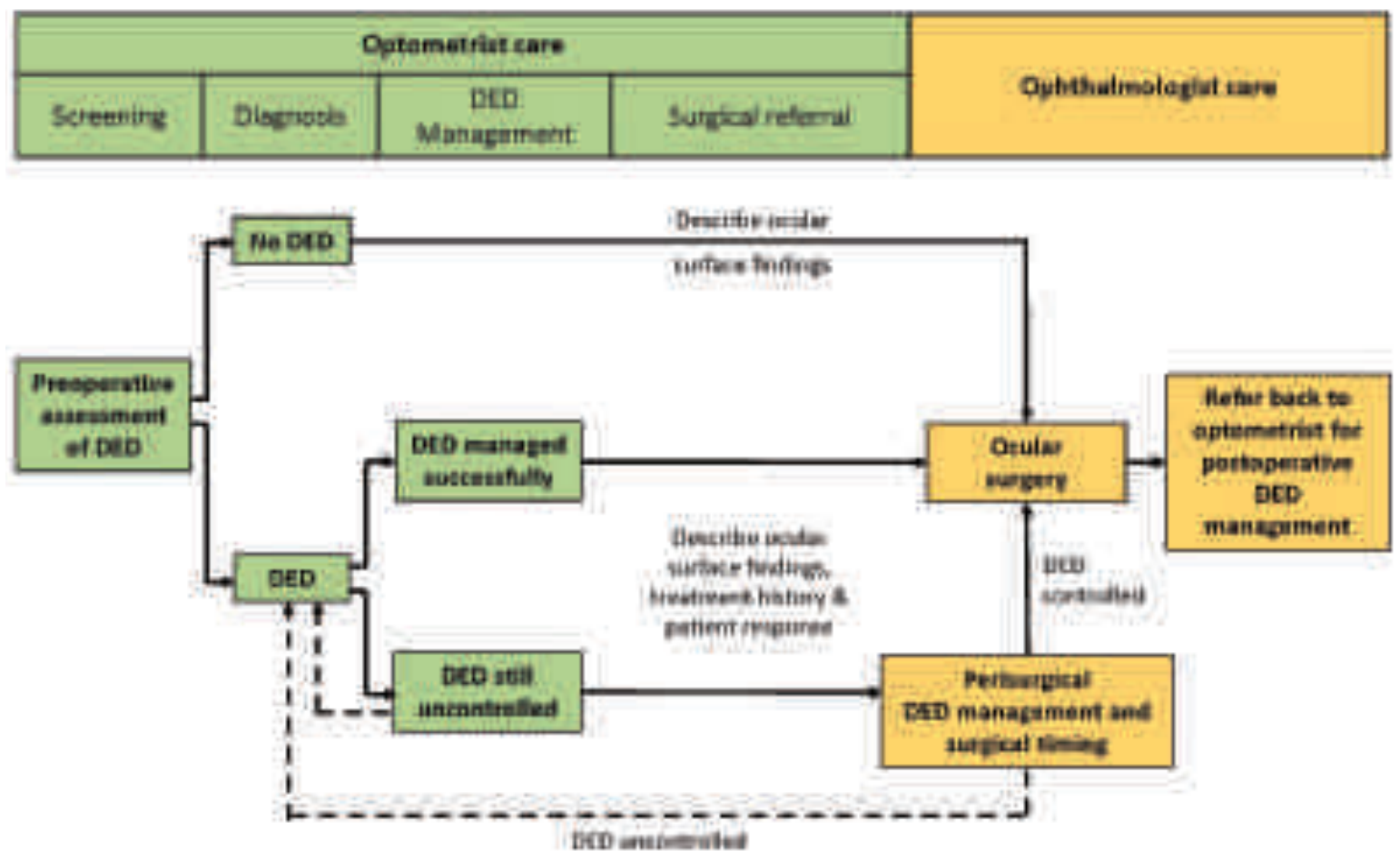
The ophthalmologist will schedule surgery if the ocular surface is stable and healthy or will refer the patient back to the optometrist for ongoing or enhanced DED treatment. In some cases, the optometrist may request that the ophthalmologist assume care of the patient, including management of the ocular surface/cornea. Following surgery, it is generally appropriate for ongoing DED care to shift back to the optometrist, unless recalcitrant disease or complications manifest that require secondary or tertiary intervention. In a return report, the surgeon should describe the nature and outcomes of the procedure, as well as any changes that may have been made for postsurgical DED management, including changes in prescription and non-prescription topical treatments.

\section{CONCLUSIONS}

DED is a chronic inflammatory disorder that optometrists and ophthalmologists encounter on a routine basis. It should be assessed and appropriately treated in all patients. The principles of DED treatment are similar whether or not ocular surgery is being considered and should follow the recommendations of the 2014 Canadian Dry Eye Disease Guidelines. ${ }^{8}$ However, DED management is particularly important before and after certain surgical procedures, specifically refractive and cataract surgery, because uncontrolled DED may place the patient at risk of less-than-optimal surgical outcomes and, conversely, surgery commonly induces or exacerbates DED. Surgery should therefore be delayed until the ocular surface has been stabilized and an adequate and appropriate tear film is restored, to the maximum extent possible. As outlined in Figure 1, anti-inflammatory treatment for patients with pre-existing chronic DED should be initiated before surgery and maintained for some months afterwards. Consensus recommendations for the management of perisurgical DED are shown in Table 4 .

Table 4: Consensus recommendations for the management of perisurgical DED

Recommendation 1. For patients with suspected ocular neuropathic pain, it is important to identify associated conditions, such as non-ocular neuropathic pain, depression, anxiety, and sleep disorders.

Recommendation 2. Depending on frequency of use, preservative-free formulations of medicated and non-medicated topical products should be considered for use before and after ocular surgery.

Recommendation 3. Independent of self-reported eye discomfort, patients undergoing cataract surgery should be assessed for signs and symptoms of DED.

Recommendation 4 . The ocular surface should be optimized prior to cataract surgery, to increase the accuracy and precision of preoperative biometry and to improve postoperative comfort and visual functioning.

Recommendation 5. Patients with pre-existing symptomatic or asymptomatic DED should be considered for treatment with anti-inflammatory agents prior to surgery, to prevent exacerbation of symptoms.

Recommendation 6. A dedicated DED assessment should be conducted as part of work-up in all patients being considered for refractive surgery.

Recommendation 7. Signs and symptoms of chronic DED, including mild DED, should be evaluated and managed in all candidates for ocular surgery.

Recommendation 8. Patients with ocular-surface staining, tear-film instability, or other signs of DED should be counselled about the risk of exacerbation of DED following ocular surgery.

Recommendation 9. Irrespective of any prior history of DED, patients undergoing ocular surgery should be counselled that DED symptoms can occur following the procedure. 
Efficient perisurgical care for patients with DED requires that optometrists and ophthalmologists co-manage the condition and communicate effectively with one another and provide consistent messages to the patient about the ocular-surface findings, risks, and response to treatment. Checklists describing best practices for correspondence between the surgeon and the optometrist are provided in Supplementary Table 1.

Supplementary Table 1:DED-related information to include in cross-referrals between optometrists and ophthalmologists

\begin{tabular}{|c|c|c|}
\hline & Optometrist to ophthalmologist & Ophthalmologist back to optometrist \\
\hline 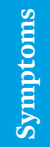 & - Sensation $\begin{array}{l}\text { Indicate } \\
\text { timeline }\end{array}$ & $\begin{array}{l}\text { - Sensation } \\
\text { - Vision } \\
\text { - Description of recent surgical procedure } \\
\text { - Outcomes (include postsurgical refraction if done) }\end{array}$ \\
\hline 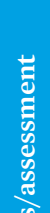 & \multicolumn{2}{|c|}{$\begin{array}{l}\text { Findings } \\
\text { - Symptom score (questionnaire) } \\
\text { - Tear stability (NIBUT/TBUT) } \\
\text { - } \text { Corneal (NaFl) and conjunctival staining (LG) } \\
\text { - Meibomian gland function } \\
\text { - } \text { Other testing (e.g., tear osmolarity) }\end{array}$} \\
\hline 总 & \multicolumn{2}{|c|}{$\begin{array}{l}\text { Description of DED/ocular surface } \\
\text { - Severity } \\
\text { - Currently stable? (if Yes, how long since stability achieved?) }\end{array}$} \\
\hline 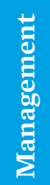 & $\begin{array}{l}\text { - Current and past treatments } \\
\text { - Tolerability issues, if any } \\
\text { - Adherence history }\end{array}$ & $\begin{array}{l}\text { Any postsurgical changes made to ocular-surface } \\
\text { management, including non-prescription products? } \\
\text { (if Yes, specify products and dosing) }\end{array}$ \\
\hline 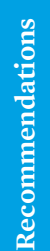 & $\begin{array}{l}\text { - Should the patient be scheduled } \\
\text { for the earliest possible surgery? } \\
\text { If No, what therapeutic milestones } \\
\text { should be achieved before scheduling } \\
\text { surgery? }\end{array}$ & $\begin{array}{l}\text { - Instructions for postsurgical care } \\
\text { - Next scheduled clinic visit or returning to optometrist's } \\
\text { care or co-management } \\
\text { - Patient's goals for vision }\end{array}$ \\
\hline
\end{tabular}

\section{ACKNOWLEDGMENTS}

This work was supported by an unrestricted grant from Allergan Inc.

\section{DISCLOSURES}

J Ashkenas received support from Allergan Canada through SCRIPT (Toronto, Canada) for his participation in this project.

E Bitton has received honoraria and/or funding for the past 3 years from Akorn, ALCON, Allergan, American Academy of Optometry, Canadian Association of Optometry, COETF, CooperVision, Labtician, I-Med Pharma Inc., Jobson Publishing, McCann Medical, Optician Journal, Novartis, Orimed, Santen, Shire, and TBWA World Health. B Caffery has received consulting fees over the past three years from Santen, Shire, Allergan, Novartis, Alcon, and Labtician.

J-S Dufour has receive honoraria over the past three years from Allergan, Novartis, and Shire.

PM Karpecki has received consulting fees from Akorn, AMO/JJV, Alcon, Allergan, B+L, Blephex, BVI, BioTissue, Bruder Healthcare, Eyegate, Focus Labs, Oculus, OcuSoft, Shire, Rendia, TearLab, TearScience, and Zeiss.

L Michaud has received honoraria and/or funding over the past three years from Alcon, Allergan, COETF, Cooper Vision, Johnson \& Johnson Vision Care, Valeant, Blanchard Labs, Genzyme, Shire, Knights Ophthalmics, and Santen. P Neumann has received funding over the past three years from Allergan Canada for speaking and consulting. V Pegado has no funding relationships to disclose.

L Racine has received consulting honoraria from Allergan, Bausch and Lomb, Johnson and Johnson Vision, Shire, Santen, and Valeant.

A Webber has received consulting and speaker fees over the past three years from Allegan, Bausch and Lomb, and Shire. 


\section{REFERENCES}

1. Smith JA, Albeitz J, Begley C et al. The Epidemiology of Dry Eye Disease: Report of the Epidemiology Subcommittee of the International Dry Eye WorkShop (2007). Ocul Surf 2007; 5(2): 93-107.

2. Moss SE, Klein R, Klein BE. Incidence of dry eye in an older population. Arch Ophthalmol 2004; 122(3): 369-73.

3. Sagdik HM, Ugurbas SH, Can M et al. Tear film osmolarity in patients with diabetes mellitus. Ophthalmic Res 2013; 50(1): 1-5.

4. Begley CG, Caffery B, Nichols KK, Chalmers R. Responses of contact lens wearers to a dry eye survey. Optom Vis Sci 2000; 77(1): 40-6.

5. Schaumberg DA, Sullivan DA, Dana MR. Epidemiology of dry eye syndrome. Adv Exp Med Biol 2002; 506(Pt B): 989-98.

6. Vivino FB, Carsons SE, Foulks G et al. New treatment guidelines for Sjogren's Disease. Rheum Dis Clin North Am 2016; 42(3): 531-51.

7. Lekskul M, Fracht HU, Cohen EJ et al. Nontraumatic corneal perforation. Cornea 2000; 19: 3-319.

8. Prokopich C, Bitton E, Caffery B et al. Screening, Diagnosis and Management of Dry Eye Disease: Practical Guidelines for Canadian Optometrists. Can J Optometry 2014; 76 (Suppl 1): 1-31.

9. Aragona P, Aguennouz M, Rania L et al. Matrix metalloproteinase 9 and transglutaminase 2 expression at the ocular surface in patients with different forms of dry eye disease. Ophthalmology 2015; 122(1): 62-71.

10. Bron AJ, Tomlinson A, Foulks GN et al. Rethinking dry eye disease: a perspective on clinical implications. Ocul Surf 2014; 12(2 Suppl): S1-31.

11. Foster CS. Dry Eye Syndrome (Keratoconjunctivitis Sicca) Medication 2016. http://emedicine.staging.medscape.com/article/1210417medication. Accessed January 2017.

12. Holland EJ, Luchs J, Karpecki PM et al. Lifitegrast for the treatment of dry eye disease: Results of a phase iii, randomized, doublemasked, placebo-controlled trial (OPUS-3). Ophthalmology 2017; 124(1): 53-60.

13. Sheppard JD, Torkildsen GL, Lonsdale JD et al. Lifitegrast ophthalmic solution $5.0 \%$ for treatment of dry eye disease: results of the OPUS-1 phase 3 study. Ophthalmology 2014; 121(2): 475-83.

14. Tauber J, Karpecki P, Latkany R et al. Lifitegrast ophthalmic solution $5.0 \%$ versus placebo for treatment of dry eye disease: Results of the randomized phase III OPUS-2 study. Ophthalmology 2015; 122(12): 2423-31.

15. Baudouin C, Figueiredo FC, Messmer EM et al. A randomized study of the efficacy and safety of $0.1 \%$ cyclosporine A cationic emulsion in treatment of moderate to severe dry eye. Eur J Ophthalmol 2017; 27(5): 520-30

16. Leonardi A, Van Setten G, Amrane M et al. Efficacy and safety of $0.1 \%$ cyclosporine A cationic emulsion in the treatment of severe dry eye disease: a multicenter randomized trial. Eur J Ophthalmol 2016; 26(4): 287-96.

17. Robert PY, Cochener B, Amrane M et al. Efficacy and safety of a cationic emulsion in the treatment of moderate to severe dry eye disease: a randomized controlled study. Eur J Ophthalmol 2016; 26(6): 546-55.

18. Cetinkaya S, Mestan E, Acir NO et al. The course of dry eye after phacoemulsification surgery. BMC Ophthalmol 2015; 15: 68.

19. Cho YK, Kim MS. Dry eye after cataract surgery and associated intraoperative risk factors. Korean J Ophthalmol 2009; 23(2): 65-73.

20. Kasetsuwan N, Satitpitakul V, Changul T, Jariyakosol S. Incidence and pattern of dry eye after cataract surgery. PLoS One 2013; 8(11): e78657.

21. Turu L, Alexandrescu C, Stana D, Tudosescu R. Dry eye disease after LASIK. J Med Life 2012; 5(1): 82-4.

22. McMonnies CW. The potential role of neuropathic mechanisms in dry eye syndromes. J Optom 2017; 10(1): 5-13.

23. Belmonte C, Nichols JJ, Cox SM et al. TFOS DEWS II pain and sensation report. Ocul Surf 2017; 15(3): 404-37.

24. Rosenthal P, Borsook D. Ocular neuropathic pain. Br J Ophthalmol 2016; 100(1): 128-34.

25. Trattler W, Reilly C, Goldberg D et al. Cataract and Dry Eye: Prospective Health Assessment of Cataract Patients Ocular Surface Study. Proceedings of the American Society of Cataract and Refractive Surgery; San Diego, CA; 2011.

26. Arciniega JC, Wojtowicz JC, Mohamed EM, McCulley JP. Changes in the evaporation rate of tear film after digital expression of Meibomian glands in patients with and without dry eye. Cornea 2011; 30(8): 843-7.

27. Lemp MA, Baudouin C, Baum J et al. The Definition and Classification of Dry Eye Disease: Report of the Definition and Classification Subcommittee of the International Dry Eye WorkShop (2007). Ocul Surf 2007; 5(2): 75-92.

28. Denoyer A, Rabut G, Baudouin C. Tear film aberration dynamics and vision-related quality of life in patients with dry eye disease. Ophthalmology 2012; 119(9): 1811-8.

29. Koh S. Mechanisms of visual disturbance in dry eye. Cornea 2016; 35 Suppl 1: S83-8.

30. Koh S, Maeda N, Hirohara Y et al. Serial measurements of higherorder aberrations after blinking in patients with dry eye. Invest Ophthalmol Vis Sci 2008; 49(1): 133-8.

31. Montes-Mico R, Caliz A, Alio JL. Changes in ocular aberrations after instillation of artificial tears in dry-eye patients. J Cataract Refract Surg 2004; 30(8): 1649-52.

32. Ambrosio R, Jr., Tervo T, Wilson SE. LASIK-associated dry eye and neurotrophic epitheliopathy: pathophysiology and strategies for prevention and treatment. J Refract Surg 2008; 24(4): 396-407.

33. Chao C, Golebiowski B, Stapleton F. The role of corneal innervation in LASIK-induced neuropathic dry eye. Ocul Surf 2014; 12(1): 32-45.

34. Nettune GR, Pflugfelder SC. Post-LASIK tear dysfunction and dysesthesia. Ocul Surf 2010; 8(3): 135-45.

35. Liu H, Thibos L, Begley CG, Bradley A. Measurement of the time course of optical quality and visual deterioration during tear breakup. Invest Ophthalmol Vis Sci 2010; 51(6): 3318-26.

36. van Landingham SW, West SK, Akpek EK et al. Impact of dry eye on reading in a population-based sample of the elderly: the Salisbury Eye Evaluation. Br J Ophthalmol 2014; 98(5): 639-44.

37. Deschamps N, Ricaud X, Rabut G et al. The impact of dry eye disease on visual performance while driving. Am J Ophthalmol 2013; 156(1): 184-9 e183.

38. De Paiva CS, Harris LD, Pflugfelder SC. Keratoconus-like topographic changes in keratoconjunctivitis sicca. Cornea 2003; 22(1): 22-4.

39. Epitropoulos AT, Matossian C, Berdy GJ et al. Effect of tear osmolarity on repeatability of keratometry for cataract surgery planning. J Cataract Refract Surg 2015; 41(8): 1672-7.

40. Kohlhaas M. Corneal sensation after cataract and refractive surgery. J Cataract Refract Surg 1998; 24(10): 1399-409.

41. Nariani A, Gupta P. Dry eye and refractive surgery outcomes. Curr Ophthalmol Rep 2016; 4: 8-14.

42. Yokoi N, Uchino M, Uchino Y et al. Importance of tear film instability in dry eye disease in office workers using visual display terminals: the Osaka study. Am J Ophthalmol 2015; 159(4): 748-54.

43. Gupta P. Cataract surgery in patients with Meibomian gland dysfunction. Cataract Refract Surg Today 2015: 77-8.

44. Potvin R. Cataracts in Canada: Introduction. Can J Optometry 2015; 77 (Suppl. 1): 4-6.

45. Canadian Association of Optometrists. Cataracts surgery in Canada: What you need to know according to the Canadian Journal of Optometry 2015. https:/opto.ca/cataracts-surgery-in-canada-whatyou-need-to-know-according-to-the-canadian-journal-of-optometry. Accessed January 2017.

46. Hatch WV, Campbell Ede L, Bell CM et al. Projecting the growth of cataract surgery during the next 25 years. Arch Ophthalmol 2012; 130(11): 1479-81.

47. Donnenfeld ED, Solomon R, Roberts CW et al. Cyclosporine $0.05 \%$ to improve visual outcomes after multifocal intraocular lens implantation. J Cataract Refract Surg 2010; 36(7): 1095-100.

48. Solomon R, Donnenfeld ED. Refractive Intraocular Lenses: Multifocal and Phakic IOLs. Int Ophthalmol Clin 2006; 46: 123-43.

49. Kuroda T, Fujikado T, Maeda N et al. Wavefront analysis of higherorder aberrations in patients with cataract. J Cataract Refract Surg 2002; 28(3): 438-44.

50. Rocha KM, Nose W, Bottos K et al. Higher-order aberrations of agerelated cataract. J Cataract Refract Surg 2007; 33(8): 1442-6.

51. Zetterberg M, Celojevic D. Gender and cataract--the role of estrogen. Curr Eye Res 2015; 40(2): 176-90.

52. Johnston J. The cataract patient is a dry eye patient. Rev Cornea Contact Lenses 2015. http://connection.ebscohost.com/c/articles/112930642/cataract-patient-dry-eye-patient. Accessed January 2017. 
53. Bron AJ, Abelson MB, Ousler G et al. Methodologies to diagnose and monitor dry eye disease: Report of the Diagnostic Methodology Subcommittee of the International Dry Eye WorkShop. Ocul Surf 2007; 5(2): 108-52.

54. Kim P, Plugfelder S, Slomovic AR. Top 5 pearls to consider when implanting advanced-technology IOLs in patients with ocular surface disease. Int Ophthalmol Clin 2012; 52(2): 51-8.

55. Li XM, Hu L, Hu J, Wang W. Investigation of dry eye disease and analysis of the pathogenic factors in patients after cataract surgery. Cornea 2007; 26(9 Suppl 1): S16-20.

56. Jiang D, Xiao X, Fu T et al. Transient tear film dysfunction after cataract surgery in diabetic patients. PLoS One 2016; 11(1): e0146752.

57. Mencucci R, Boccalini C, Caputo R, Favuzza E. Effect of a hyaluronic acid and carboxymethylcellulose ophthalmic solution on ocular comfort and tear-film instability after cataract surgery. J Cataract Refract Surg 2015; 41(8): 1699-704.

58. Sanchez MA, Arriola-Villalobos P, Torralbo-Jimenez P et al. The effect of preservative-free HP-Guar on dry eye after phacoemulsification: a flow cytometric study. Eye (Lond) 2010; 24(8): 1331-7.

59. Jee D, Park M, Lee HJ et al. Comparison of treatment with preservative-free versus preserved sodium hyaluronate $0.1 \%$ and fluorometholone $0.1 \%$ eyedrops after cataract surgery in patients with preexisting dry-eye syndrome. J Cataract Refract Surg 2015; 41(4): 756-63.

60. Chen M, Gong L, Sun X et al. A comparison of cyclosporine $0.05 \%$ ophthalmic emulsion versus vehicle in Chinese patients with moderate to severe dry eye disease: an eight-week, multicenter, randomized, double-blind, parallel-group trial. J Ocul Pharmacol Ther 2010; 26(4): 361-6.

61. Rao SN. Topical cyclosporine $0.05 \%$ for the prevention of dry eye disease progression. J Ocul Pharmacol Ther 2010; 26(2): 157-64.

62. Rao SN. Reversibility of dry eye deceleration after topical cyclosporine $0.05 \%$ withdrawal. J Ocul Pharmacol Ther 2011; 27(6): 603-9.

63. Chung YW, Oh TH, Chung SK. The effect of topical cyclosporine $0.05 \%$ on dry eye after cataract surgery. Korean J Ophthalmol 2013 27(3): 167-71.

64. Lee JH, Song IS, Kim KL, Yoon SY. Effectiveness and optical quality of topical 3.0\% Diquafosol versus $0.05 \%$ Cyclosporine A in dry eye patients following cataract surgery. J Ophthalmol 2016; 8150757.

65. Hamada S, Moore TC, Moore JE et al. Assessment of the effect of cyclosporine-A $0.05 \%$ emulsion on the ocular surface and corneal sensation following cataract surgery. Cont Lens Anterior Eye 2016; 39(1): 15-9.

66. Ursea R, Purcell TL, Tan BU et al. The effect of cyclosporine A (Restasis) on recovery of visual acuity following LASIK. J Refract Surg 2008; 24(5): 473-6.

67. McGhee CN, Orr D, Kidd B et al. Psychological aspects of excimer laser surgery for myopia: reasons for seeking treatment and patient satisfaction. Br J Ophthalmol 1996; 80(10): 874-9.

68. Toda I, Asano-Kato N, Hori-Komai Y, Tsubota K. Ocular surface treatment before laser in situ keratomileusis in patients with severe dry eye. J Refract Surg 2004; 20(3): 270-5.

69. Xie W. Recent advances in laser in situ keratomileusis-associated dry eye. Clin Exp Optom 2016; 99(2): 107-12.

70. Albietz JM, Lenton LM. Management of the ocular surface and tear film before, during, and after laser in situ keratomileusis. J Refract Surg 2004; 20(1): 62-71.

71. Torricelli AA, Bechara SJ, Wilson SE. Screening of refractive surgery candidates for LASIK and PRK. Cornea 2014; 33(10): 1051-5.

72. Garcia-Zalisnak D, Nash D, Yeu E. Ocular surface diseases and corneal refractive surgery. Curr Opin Ophthalmol 2014; 25(4): 264-9.

73. Torricelli AA, Santhiago MR, Wilson SE. Topical cyclosporine a treatment in corneal refractive surgery and patients with dry eye. J Refract Surg 2014; 30(8): 558-64.

74. Denoyer A, Landman E, Trinh L et al. Dry eye disease after refractive surgery: comparative outcomes of small incision lenticule extraction versus LASIK. Ophthalmology 2015; 122(4): 669-76.

75. Wang B, Naidu RK, Chu R et al. Dry Eye Disease following refractive surgery: A 12-month follow-up of SMILE versus FS-LASIK in high myopia. J Ophthalmol 2015; 132417

76. Bower KS, Sia RK, Ryan DS et al. Chronic dry eye in photorefractive keratectomy and laser in situ keratomileusis: Manifestations, incidence, and predictive factors. J Cataract Refract Surg 2015; 41(12): 2624-34

77. Albietz JM, Lenton LM, McLennan SG. Effect of laser in situ keratomileusis for hyperopia on tear film and ocular surface. J Refract
Surg 2002; 18(2): 113-23.

78. Esquenazi S. Five-year follow-up of laser in situ keratomileusis for hyperopia using the Technolas Keracor $117 \mathrm{C}$ excimer laser. J Refract Surg 2004; 20(4): 356-63.

79. Goto T, Zheng X, Klyce SD et al. Evaluation of the tear film stability after laser in situ keratomileusis using the tear film stability analysis system. Am J Ophthalmol 2004; 137(1): 116-20.

80. Peyman GA, Sanders DR, Batlle JF et al. Cyclosporine $0.05 \%$ ophthalmic preparation to aid recovery from loss of corneal sensitivity after LASIK. J Refract Surg 2008; 24(4): 337-43.

81. Albietz JM, Lenton LM, McLennan SG. Chronic dry eye and regression after laser in situ keratomileusis for myopia. J Cataract Refract Surg 2004; 30(3): 675-84.

82. Sall K, Stevenson OD, Mundorf TK, Reis BL. Two multicenter, randomized studies of the efficacy and safety of cyclosporine ophthalmic emulsion in moderate to severe dry eye disease. CsA Phase 3 Study Group. Ophthalmology 2000; 107(4): 631-9.

83. Salib GM, McDonald MB, Smolek M. Safety and efficacy of cyclosporine $0.05 \%$ drops versus unpreserved artificial tears in dry-eye patients having laser in situ keratomileusis. J Cataract Refract Surg 2006; 32(5): 772-8.

84. Sheppard JD, Jr., Singh R, McClellan AJ et al. Long-term supplementation with n-6 and n-3 PUFAs improves moderate-to-severe keratoconjunctivitis sicca: A randomized double-blind clinical trial. Cornea 2013; 32(10): 1297-304.

85. Zhu W, Wu Y, Li G et al. Efficacy of polyunsaturated fatty acids for dry eye syndrome: a meta-analysis of randomized controlled trials. Nutr Rev 2014; 72(10): 662-71.

86. Khalil MB, Latkany RA, Speaker MG, Yu G. Effect of punctal plugs in patients with low refractive errors considering refractive surgery. J Refract Surg 2007; 23(5): 467-71.

87. Di Pascuale MA, Liu TS, Trattler W, Tseng SC. Lipid tear deficiency in persistent dry eye after laser in situ keratomileusis and treatment results of new eye-warming device. J Cataract Refract Surg 2005; 31(9): 1741-9.

88. Schaumberg DA, Nichols JJ, Papas EB et al. The international workshop on Meibomian gland dysfunction: report of the subcommittee on the epidemiology of, and associated risk factors for, MGD. Invest Ophthalmol Vis Sci 2011; 52(4): 1994-2005.

89. Abelson M, Rosenthal P, McLaughlin J. Neuropathic pain: The artifice of dry eye. Review of Ophthalmology 2016; 23(1): 1-3.

90. Belmonte C, Acosta MC, Merayo-Lloves J, Gallar J. What causes eye pain? Curr Ophthalmol Rep 2015; 3(2): 111-21.

91. Galor A, Batawi H, Felix ER et al. Incomplete response to artificial tears is associated with features of neuropathic ocular pain. $\mathrm{Br} \mathrm{J}$ Ophthalmol 2016; 100(6): 745-9.

92. Crane AM, Levitt RC, Felix ER et al. Patients with more severe symptoms of neuropathic ocular pain report more frequent and severe chronic overlapping pain conditions and psychiatric disease. Br J Ophthalmol 2017; 101(2): 227-31.

93. Shtein RM, Harper DE, Pallazola V et al. Discordant dry eye disease (An American Ophthalmological Society Thesis). Trans Am Ophthalmol Soc 2016; 114: T4.

94. Baudouin C. Side effects of antiglaucomatous drugs on the ocular surface. Curr Opin Ophthalmol 1996; 7(2): 80-6.

95. Baudouin C, Renard JP, Nordmann JP et al. Prevalence and risk factors for ocular surface disease among patients treated over the long term for glaucoma or ocular hypertension. Eur J Ophthalmol 2012: 0.

96. Conlon R, Saheb H, Ahmed, II. Glaucoma treatment trends: a review. Can J Ophthalmol 2017; 52(1): 114-24.

97. Pisella PJ, Pouliquen P, Baudouin C. Prevalence of ocular symptoms and signs with preserved and preservative free glaucoma medication. Br J Ophthalmol 2002; 86(4): 418-23.

98. Garcia-Feijoo J, Sampaolesi JR. A multicenter evaluation of ocular surface disease prevalence in patients with glaucoma. Clin Ophthalmol 2012; 6: 441-6.

99. Leung EW, Medeiros FA, Weinreb RN. Prevalence of ocular surface disease in glaucoma patients. J Glaucoma 2008; 17(5): 350-5.

100. Ramli N, Supramaniam G, Samsudin A et al. Ocular surface disease in glaucoma: Effect of polypharmacy and preservatives. Optom Vis Sci 2015; 92(9): e222-6.

101. Warcoin E, Clouzeau C, Roubeix C et al. Hyperosmolarity and benzalkonium chloride differently stimulate inflammatory markers in conjunctiva-derived epithelial cells in vitro. Ophthalmic Res 2017; 58(1): 40-8. 
102. Broadway DC, Grierson I, Sturmer J, Hitchings RA. Reversal of topical antiglaucoma medication effects on the conjunctiva. Arch Ophthalmol 1996; 114(3): 262-7.

103. Kersey JP, Broadway DC. Corticosteroid-induced glaucoma: a review of the literature. Eye (Lond) 2006; 20(4): 407-16.

104. Skalicky SE, Goldberg I, McCluskey P. Ocular surface disease and quality of life in patients with glaucoma. Am J Ophthalmol 2012; 153(1): 1-9 e2.

105. Fechtner RD, Godfrey DG, Budenz D et al. Prevalence of ocular surface complaints in patients with glaucoma using topical intraocular pressure-lowering medications. Cornea 2010; 29(6): 618-21.

106. Saini M, Dhiman R, Dada T et al. Topical cyclosporine to control ocular surface disease in patients with chronic glaucoma after longterm usage of topical ocular hypotensive medications. Eye (Lond) 2015; 29(6): 808-14.

107. Batra R, Tailor R, Mohamed S. Ocular surface disease exacerbated glaucoma: optimizing the ocular surface improves intraocular pressure control. J Glaucoma 2014; 23(1): 56-60.

108. de Jong C, Stolwijk T, Kuppens E et al. Topical timolol with and without benzalkonium chloride: epithelial permeability and autofluorescence of the cornea in glaucoma. Graefes Arch Clin Exp Ophthalmol 1994; 232(4): 221-4.

109. Gayton JL, Van Der Karr M, Sanders V. Combined cataract and glaucoma surgery: trabeculectomy versus endoscopic laser cycloablation. J Cataract Refract Surg 1999; 25(9): 1214-9.

110. Tanbakouee E, Ghoreishi M, Aghazadeh-Amiri M et al. Photorefractive keratectomy for patients with preoperative low Schirmer test value. J Curr Ophthalmol 2016; 28(4): 176-80.

111. Al-Swailem SA. Graft failure: II. Ocular surface complications. Int Ophthalmol 2008; 28(3): 175-89.

112. Sheppard JJ. Prevalence of dry eye in planned penetrating or endothelial keratoplasty. abstractsnet.com/handouts/0225_Dry_eye_ prevalence_WCC_2015.pptx. Accessed January 2017.

113. Tan DT, Dart JK, Holland EJ, Kinoshita S. Corneal transplantation. Lancet 2012; 379(9827): 1749-61.

114. Shousha MA, Yoo SH, Kymionis GD et al. Long-term results of femtosecond laser-assisted sutureless anterior lamellar keratoplasty. Ophthalmology 2011; 118(2): 315-23.
115. Li M, Zhang M, Lin Y et al. Tear function and goblet cell density after pterygium excision. Eye (Lond) 2007; 21: 224-8.

116. Chhadva P, Alexander A, McClellan AL et al. The impact of conjunctivochalasis on dry eye symptoms and signs. Invest Ophthalmol Vis Sci 2015; 56(5): 2867-71.

117. Di Pascuale MA, Espana EM, Kawakita T, Tseng SC. Clinical characteristics of conjunctivochalasis with or without aqueous tear deficiency. Br J Ophthalmol 2004; 88(3): 388-92.

118. Hara S, Kojima T, Ishida R et al. Evaluation of tear stability after surgery for conjunctivochalasis. Optom Vis Sci 2011; 88(9): 1112-8.

119. Acera A, Vecino E, Duran JA. Tear MMP-9 levels as a marker of ocular surface inflammation in conjunctivochalasis. Invest Ophthalmol Vis Sci 2013; 54(13): 8285-91.

120. Yu EY, Leung A, Rao S, Lam DS. Effect of laser in situ keratomileusis on tear stability. Ophthalmology 2000; 107(12): 2131-5.

121. Saedon H, Nosek J, Phillips J et al. Ocular surface effects of repeated application of povidone iodine in patients receiving frequent intravitreal injections. Cutan Ocul Toxicol 2017: 1-4.

122. Bagheri A, Najmi H, Salim RE, Yazdani S. Tear condition following unilateral ptosis surgery. Orbit 2015; 34(2): 66-71.

123. Prischmann J, Sufyan A, Ting JY et al. Dry eye symptoms and chemosis following blepharoplasty: a 10-year retrospective review of 892 cases in a single-surgeon series. JAMA Facial Plast Surg 2013; 15(1): 39-46.

124. Saadat D, Dresner SC. Safety of blepharoplasty in patients with preoperative dry eyes. Arch Facial Plast Surg 2004; 6(2): 101-4.

125. Lee SY, Wong TT, Chua J et al. Effect of chronic anti-glaucoma medications and trabeculectomy on tear osmolarity. Eye (Lond) 2013; 27(10): 1142-50.

126. Boimer C, Birt CM. Preservative exposure and surgical outcomes in glaucoma patients: The PESO study. J Glaucoma 2013; 22(9): 730-5.

127. Li Q, Fu T, Yang J et al. Ocular surface changes after strabismus surgery with different incisions. Graefes Arch Clin Exp Ophthalmol 2015; 253(3): 431-8. 